\title{
Unfolding of the vortical amplification of the magnetic field at inward shocks of Supernova remnant Cassiopeia A
}

\section{F. Fraschetti, ${ }^{a, b, *}$ S. Katsuda, ${ }^{c}$ T. Sato, ${ }^{d}$ J. Giacalone ${ }^{b}$ and J. R. Jokipii ${ }^{b}$}

${ }^{a}$ Center for Astrophysics | Harvard \& Smithsonian, Cambridge, MA, 02138, USA

${ }^{b}$ Lunar \& Planetary Laboratory, Department of Planetary Sciences, University of Arizona, Tucson, AZ 85721, USA

${ }^{c}$ Graduate School of Science and Engineering, Saitama University, 255 Shimo-Ohkubo, Sakura, Saitama 338-8570, Japan

${ }^{d}$ RIKEN Nishina Center, 2-1 Hirosawa, Wako, Saitama 351-0198, Japan

E-mail: federico.fraschetti@cfa.harvard.edu

Observational evidence of strongly amplified magnetic fields at interstellar supernova remnant (SNR) shocks has been proposed to result from downstream small scale dynamo; however, quantitative comparison with observations of $X$-ray time variability has been not extensively explored. We present an interpretation of the time variability of the $X$-ray flux reported from a 15 years multi-epoch observational campaign of the supernova remnant Cassiopeia A by Chandra. The increase of the [4.2-6] keV non-thermal flux up to 50\% is shown quantitatively to trace the growth of the magnetic field due to vortical amplification mechanism downstream of inward shocks colliding with inner overdensities. The fast synchrotron cooling as compared with shock-acceleration time scale is consistent with the subsequent flux decrease.

$37^{\text {th }}$ International Cosmic Ray Conference (ICRC 2021)

July 12 th - 23rd, 2021

Online - Berlin, Germany

\footnotetext{
${ }^{*}$ Presenter
} 


\section{Introduction}

The year time-scale variability in the $X$-ray filaments and knots of the supernova remnant Cassiopeia $\mathrm{A}$ was associated [1] with a fast synchrotron cooling in strong magnetic field; a decline of the $X$-ray flux between 2000 and 2010 was observed with Chandra in the entire remnant's western limb [2]. High spatial resolution multi-epoch observations of Cassiopeia A have shown unprecedented evidence of an increase followed by a decrease of $X$-ray flux ([4.2-6] keV band) up to $50 \%$ in six distinct regions approximately 10 " $\times 10$ " or 15 " $\times 15$ " in size located on the west side and toward the center of the remnant [3]; such observations cover a time period of 15 years (from 2000 to 2014). The location of these regions is consistent with a high speed shock observed to move inward. Due to the young age of the remnant, such a shock is unlikely to correspond to the reverse shock, that would move outward at such evolution stage, and plausibly originated as a reflection [4] from the collision of the forward shock with an interstellar medium molecular cloud; however, evidence of large scale upstream inhomogeneities is under debate [5, 6]. The inward shock surface is likely to be corrugated to the scale of the molecular cloud or of the inhomogeneities within the shocked layer.

In [7] overdensity clumps within the expanding plasma of Cassiopeia A were shown to lead to the $[4.2-6] \mathrm{keV}$ flux increase via magnetic field amplification through vorticity generation, after crossing by the reflection inward shock. Such a process was investigated numerically $[8,9]$, analytically [10] and in laboratory experiments [11]. Figure 1 shows a cartoon illustration of the scenario proposed in [7]: the corrugated inward shock travels through the shocked layer and therein collides with density clumps, generates vorticity and amplifies the magnetic field in the downstream medium.

The induction equation for $B$ was solved in [10] to determine the MHD jump conditions at corrugated shock fronts; the vorticity generated behind the shock is related to $B$ via the small-scale dynamo process [12] leading to

$$
\left(\frac{B}{B_{0}}\right)^{2}(t)=\frac{e^{2 t / \tau}}{1-\alpha \tau\left(1-e^{2 t / \tau}\right) v_{A}^{2} / 2},
$$

where $B_{0}$ is the upstream seed magnetic strength and

$$
\tau=\frac{r}{r-1} \frac{1}{C_{r}} \frac{R_{c} \ell_{F}}{R_{c}+\ell_{F}}
$$

is the growth time-scale determined by the shock compression $r$, the curvature radius of the ripples on the forward shock surface $R_{c}$ that is expected to be comparable with the size of the overdensity clumps, the thickness of the outer clump layer where the density gradient is non-vanishing $\ell_{F}$ (corresponding to the Field length in the ISM) and the shock speed in the upstream frame $C_{r}$; $\alpha \sim 1 / R_{C} C_{r}$ describes the field back-reaction to the whirling of the fluid and $v_{A}$ is the seed field Alfvén speed. As calculated in detail in [10], the $B$-amplification occurs within the outer layer of thickness $\ell_{F}$.

\section{Flux increase}

The main assumption is that the time-variation of the non-thermal emission (assumed to be synchrotron radiation by energetic electrons) is due to the time-variation of the downstream magnetic 


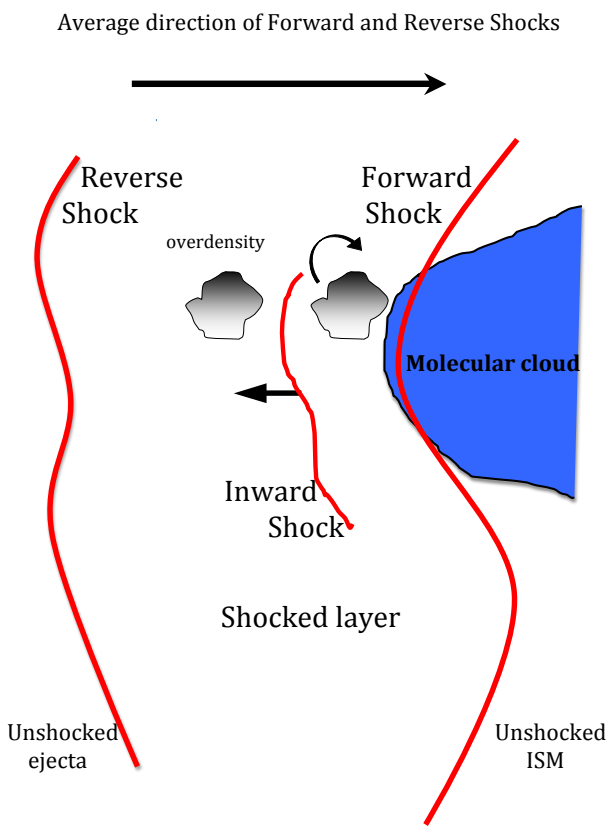

Figure 1: Cartoon illustration: the inward shock recedes into the shocked layer and crosses outward moving clumps. The arrows indicate the direction of the shocks motion in the observer frame.

field $B(t)$. For a differential energy distribution of the energetic electrons given by a simple powerlaw, it holds $d N / d \gamma=N_{0}\left(\gamma / \gamma_{0}\right)^{-p}$, where $\gamma_{0}$ is the injection electron Lorentz factor and the index $p$ is determined by the shock compression only, as predicted by the linear test-particle version of the diffusive shock acceleration model. Within such a narrow energy band more sophisticated spectral models seem unnecessary.

The total synchrotron flux at Earth from Cas A, located at distance $d$, namely $v F_{v}$, is found by folding the single electron isotropic power with $d N / d \gamma$. Assuming that the electron power is concentrated around the characteristic synchrotron energy $\epsilon_{s}=0.29\left(3 e h \gamma^{2} B\right) /\left(4 \pi m_{e} c\right)$, where $e$ is the electron charge and $h$ is the Planck constant, we recast the total flux observed at Earth as

$$
v F_{v}(\epsilon, t)=\frac{1}{4 \pi d^{2}} \frac{\sigma_{T} c}{12 \pi} \frac{N_{0}}{\mathcal{A}} \gamma_{0}^{p} \epsilon^{-\frac{p-3}{2}} B(t)^{2+\frac{p-3}{2}}
$$

where $\sigma_{T}$ is the Thomson cross-section, $c$ speed of light in vacuum and the constant $\mathcal{A}$ is defined as $\mathcal{A}=\left[0.29(3 e h) /\left(4 \pi m_{e} c\right)\right]^{-\frac{p-3}{2}}$.

In [7] we reproduce (see Fig. 2 upper panel), the observed flux change in the range [4.2 - 6] $\mathrm{keV}$ compared with the theoretical prediction (from Eq.3) for $\epsilon=5 \mathrm{keV}, d=3.4 \mathrm{kpc}$ and for distinct values of $R_{c}$. The model of the flux increase depends only on two fitting parameters, $R_{c}$ and $\ell_{F}$. The best-fit values for the time-interval [2000:2009] are $R_{C}=(1.00 \pm 0.16) \times 10^{18} \mathrm{~cm}$ and $\ell_{F}=(7.03 \pm 0.76) \times 10^{17} \mathrm{~cm}$. The error bars on $R_{c}$ and $\ell_{F}$ are likely dominated by the uncertainty on the exponential rise. In addition, the 2009 data-point belongs to the incipient decrease phase of the flux, hence is not accounted for by our analytic model. 


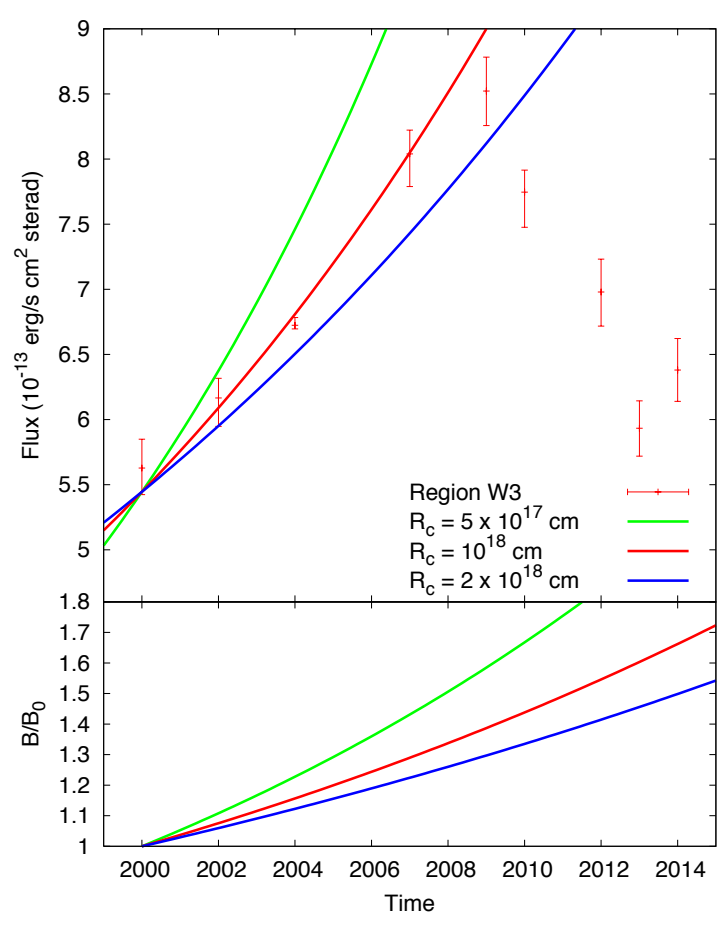

Figure 2: Upper panel: Theoretical synchrotron flux at $\epsilon=5 \mathrm{keV}$ as a function of time for distinct values of $R_{C}$ compared with Chandra $[4.2-6] \mathrm{keV}$ observations; here $\ell_{F}=7.03 \times 10^{17} \mathrm{~cm}$. The best fit is represented by the red line. Lower panel: Time-evolution of relative magnetic strength during the turbulent amplification for the three cases shown above, with the same color-labels.

The inward shocks are likely to be travelling into a hot plasma with electron temperature $\sim 2$ $\mathrm{keV}$ (see also [13]), in equilibrium with ions: narrow lines in some bright knots indicate low ion temperature within the remnant [14]. For the W3 inward shock $C_{r}=6,500 \mathrm{~km} / \mathrm{s}$ in the shocked layer frame [table 3 in ref. 3]. Comparable best-fit values of $R_{c}$ and $\ell_{F}$ for the other inward shocks reported in [3] were found by [15].

\section{Flux decrease}

The time of the peak of $v F_{v}^{[4.2-6] \mathrm{keV}}$, occurring in $\sim 2009$ for $\mathrm{W} 3$, was approximated by the ratio of the synchrotron cooling time scale, $t_{c o o l}$, to the acceleration time-scale, $t_{a c c}$. For an electron emitting synchrotron radiation in an ambient downstream magnetic field $B(t)$ at a typical photon energy $\epsilon$, it holds

$$
t_{\text {cool }}(t) \simeq(55 \mathrm{yr}) \epsilon_{k e V}^{-1 / 2} B_{0.1}(t)^{-3 / 2} .
$$

At $\epsilon_{k e V}=\epsilon / 1 \mathrm{keV}=5$, the value $t_{\text {cool }}=4$ yrs yields at a time $t^{\star}=2009$ the field $B_{0.1}\left(t^{\star}\right) \simeq$ 3.4, in the expected range for Cassiopeia A, as discussed in Ref. [1]. The best-fit $B$-field (solid line in the lower panel in Fig. 2) shows a ratio $B\left(t^{\star}\right) / B_{0} \simeq 1.35$ that leads to $B_{0(0.1)}=2.5$, with $B_{0(0.1)}=B_{0} / 0.1 \mathrm{mG}$. Such a relatively high $B_{0}$ confirms that an amplification in W3 likely took place at the inward shock prior to year 2000 , possibly via vorticity generation within the shocked layer due to the corrugation of the inward shock or an alternative mechanism [16]. 
The acceleration time-scale $t_{a c c}$ can be approximated by[17]

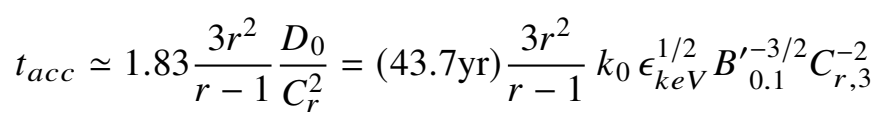

where for an isotropic upstream turbulence $D_{0}$ is the diffusion coefficient at the electron cut-off energy along the average direction of shock motion and $k_{0}$, assumed [17] to be equal upstream and downstream, is given by $k_{0}=D_{0} / D_{B}$, where $D_{B}$ is the Bohm diffusion coefficient at that energy. The departure from unity of $k_{0}$, both $k_{0}>1\left(k_{0}<1\right)$ for shocks close to quasi-parallel (or quasi-perpendicular) topology within the acceleration region, indicates that the turbulence is not dominant over the seed field [18]. Finally, the upstream field, constant in time as the amplification occurs downstream in the model presented here for the flux increase (the effect of plasma kinetic instabilities on the observed X-ray emission is neglected herein), is given by $B^{\prime}{ }_{0.1}=B^{\prime} / 0.1 \mathrm{mG}$ and $C_{r, 3}=C_{r} /(1,000 \mathrm{~km} / \mathrm{s})$. For the speed $C_{r, 3}=6,5$ at the latest $C$ andra observation, in region W3 we estimate $k_{0}=4.7$ from Eq. 3 in [3]; for $B^{\prime}{ }_{0.1}=2.5$, Eq. 5 provides $t_{a c c} \sim 42$ years, significantly greater than $t_{\text {cool }} \sim 4$ years. A cooling faster than the acceleration is consistent with the observed flux decrease. More sophisticated models for the diffusion coefficient (e.g., [19], [20]) will not substantially change this qualitative argument. An extended discussion can be found in [7]. In the analysis by [21] of a sample of 11 young SNRs, including Cas A, the maximum energy attained by accelerated particles is inferred to be higher if the time evolution of the turbulent magnetic component is included. The present work suggests that the vortical amplification downstream of shocks needs to be accounted for in the modelling of the synchrotron emission time-variability. The analysis presented herein offers a quantitative interpretation of Chandra observations in terms of magnetic field dynamics.

\section{Conclusion}

We have outlined that the $X$-ray flux increase between years 2000 and 2009 in a number of small regions close to center and in the west limb of Cassiopeia A can trace [7] the growth of the magnetic field due to vortical amplification as determined in [10]. Values of the diffusion coefficient departing from the Bohm limit, that indicate relatively weak turbulence around the shock, are also consistent with the inferred modest field amplification. We have provided [7] a qualitative argument but not a firm theoretical model for the flux decrease between years 2009 and 2014. Further analysis is warranted and high-resolution follow-up monitoring of the region W3 is encouraged. This work demonstrates that the unfolding of a dynamo process formerly theoretically identified can be not only investigated in laboratory plasma astrophysics but also observed in astrophysical systems.

\section{Acknowledgments}

The work of F.F. was supported in part by NASA under Grant No. NNX15AJ71G. F.F. also acknowledges support from Smithsonian Institution Scholarly Studies Grant No. 40488100 HH00181. The work of S.K. was partially supported by the Japan Society for the Promotion of Science KAKENHI Grant No. 16K17673 and No. $17 \mathrm{H} 02864$ and partially by Leading Initiative for Excellent Young Researchers, MEXT, Japan. T.S. was supported by the Special Postdoctoral Researchers Program in RIKEN. 


\section{References}

[1] Y. Uchiyama and F.A. Aharonian, Fast Variability of Nonthermal X-Ray Emission in Cassiopeia A: Probing Electron Acceleration in Reverse-Shocked Ejecta, 677 (2008) L105 [0803.3410].

[2] D.J. Patnaude, J. Vink, J.M. Laming and R.A. Fesen, A Decline in the Nonthermal X-ray Emission from Cassiopeia A, 729 (2011) L28 [1012 . 0243].

[3] T. Sato, S. Katsuda, M. Morii, A. Bamba, J.P. Hughes, Y. Maeda et al., X-Ray Measurements of the Particle Acceleration Properties at Inward Shocks in Cassiopeia A, 853 (2018) 46 [1710.06992].

[4] A.G. Sgro, The collision of a strong shock with a gas cloud - A model for Cassiopeia A, 197 (1975) 621.

[5] C.D. Kilpatrick, J.H. Bieging and G.H. Rieke, Interaction between Cassiopeia A and Nearby Molecular Clouds, 796 (2014) 144 [1411.4308].

[6] P. Zhou, J.-T. Li, Z.-Y. Zhang, J. Vink, Y. Chen, M. Arias et al., Molecular Gas toward Supernova Remnant Cassiopeia A, 865 (2018) 6 [1808.03058].

[7] F. Fraschetti, S. Katsuda, T. Sato, J.R. Jokipii and J. Giacalone, Vortical Amplification of the Magnetic Field at an Inward Shock of Supernova Remnant Cassiopeia A, Physical Review Letters 120 (2018) 251101 [1805 . 05951].

[8] J. Giacalone and J.R. Jokipii, Magnetic Field Amplification by Shocks in Turbulent Fluids, 663 (2007) L41.

[9] T. Inoue, R. Yamazaki, S.-i. Inutsuka and Y. Fukui, Toward Understanding the Cosmic-Ray Acceleration at Young Supernova Remnants Interacting with Interstellar Clouds: Possible Applications to RX J1713.7-3946, 744 (2012) 71 [1106.3080].

[10] F. Fraschetti, Turbulent Amplification of a Magnetic Field Driven by the Dynamo Effect at Rippled Shocks, 770 (2013) 84 [1304.4956].

[11] J. Meinecke, H.W. Doyle, F. Miniati, A.R. Bell, R. Bingham, R. Crowston et al., Turbulent amplification of magnetic fields in laboratory laser-produced shock waves, Nature Physics 10 (2014) 520.

[12] R.M. Kulsrud and S.W. Anderson, The spectrum of random magnetic fields in the mean field dynamo theory of the Galactic magnetic field, 396 (1992) 606.

[13] U. Hwang and J.M. Laming, A Chandra X-Ray Survey of Ejecta in the Cassiopeia A Supernova Remnant, 746 (2012) 130 [1111.7316].

[14] J.S. Lazendic, D. Dewey, N.S. Schulz and C.R. Canizares, The Kinematic and Plasma Properties of X-Ray Knots in Cassiopeia A from the Chandra HETGS, 651 (2006) 250 [astro-ph/0605078]. 
[15] M. Rassel and F. Fraschetti, Amplification of magnetic field downstream of inward shocks in supernova remnant Cassiopeia A, in American Astronomical Society Meeting Abstracts \#233, vol. 233 of American Astronomical Society Meeting Abstracts, p. 410.03, Jan, 2019.

[16] S. Xu and A. Lazarian, Small-scale turbulent dynamo in astrophysical environments: nonlinear dynamo and dynamo in a partially ionized plasma, Reviews of Modern Plasma Physics 5 (2021) 2.

[17] E. Parizot, A. Marcowith, J. Ballet and Y.A. Gallant, Observational constraints on energetic particle diffusion in young supernovae remnants: amplified magnetic field and maximum energy, 453 (2006) 387 [astro-ph/0603723].

[18] F. Fraschetti and J. Giacalone, Early-time Velocity Autocorrelation for Charged Particles Diffusion and Drift in Static Magnetic Turbulence, 755 (2012) 114 [1206.6494].

[19] J. Giacalone, Cosmic-Ray Transport and Interaction with Shocks, 176 (2013) 73.

[20] F. Fraschetti and J.R. Jokipii, Time-dependent Perpendicular Transport of Fast Charged Particles in a Turbulent Magnetic Field, 734 (2011) 83 [1104. 0955].

[21] N. Tsuji, Y. Uchiyama, D. Khangulyan and F. Aharonian, Systematic Study of Acceleration Efficiency in Young Supernova Remnants with Nonthermal X-Ray Observations, 907 (2021) 117 [2012.01047]. 